C 1996 IEEE. Personal use of this material is permitted. However, permission to reprint/republish this material

for advertising or promotional purposes or for creating new collective works for resale or redistribution to servers

or lists, or to reuse any copyrighted component of this work in other works must be obtained from the IEEE.

\title{
Simulations of Sawtooth Instability
}

\author{
R. BAARTMAN and M. D'YACHKOV, \\ TRIUMF, 4004 Wesbrook Mall, Vancouver, B.C. Canada, V6T 2A3
}

\begin{abstract}
The equilibrium self-consistent distribution of particles in a high intensity electron synchrotron can be found using the Haissinski equation and the wake ßeld. At some threshold intensity the bunch becomes unstable. However, radiation damping causes the particles to be con®ned and the instability does not necessarily cause loss of particles.

It was observed in simulations with a very simple wake ${ }^{\circledR e l d}$ and short bunches, that energy spread and bunch length oscillate in a sawtooth fashion. We ${ }^{\circledR}$ nd that this is due to the doublepeaked nature of the stationary distribution. Over many synchrotron oscillations, particles diffuse from the head peak to the tail to the point where the tail peak becomes as large as the head. The two resulting sub-bunches then collapse together in less than one synchrotron oscillation, causing a net blow-up in emittance. Radiation damping reduces the emittance and diffusion begins again.
\end{abstract}

\section{Introduction}

A so-called 'sawtooth' instability has been observed in the SLC damping rings [1] and there is evidence that it has been observed in other electron synchrotrons as well [2]. This instability appears as a periodic fast blow-up in bunch length, followed by damping. We studied the origins of this effect using multi-particle tracking. Rather than trying to describe an actual machine, as was done by Bane and Oide [3], we simplißed the model to determine which features of the wake ®eld lead to a sawtooth behaviour.

We describe the results of the simulation and then give a qualitative discussion of the origins of the instability.

\section{Numerical Simulations}

\section{A. Model}

To simulate the electron's motion in a synchrotron we use a standard multi-particle tracking scheme [4]. The beam is represented by $N$ macroparticles each with phase and energy coordinates $\left(z_{i}, e_{i}\right)$. These coordinates are recalculated every turn according to the following equations.

$$
\begin{aligned}
\Delta e_{i} & =-\frac{2 T_{0}}{\tau_{e}} e_{i}+2 \sigma_{e 0} \sqrt{\frac{T_{0}}{\tau_{e}}} r_{i}+V_{\mathrm{rf}}^{\prime} z_{i}+V_{\mathrm{ind}}\left(z_{i}\right) \\
\Delta z_{i} & =\frac{\alpha c T_{0}}{E_{0}}\left(e_{i}+\Delta e_{i}\right)
\end{aligned}
$$

$T_{0}$ is the revolution period, $\tau_{e}$ is the damping time, $\sigma_{e 0}$ the rms energy spread in the absence of a wake, $V_{\mathrm{rf}}^{\prime}$ the slope of the rf voltage, $\alpha$ is the compaction factor, and $E_{0}$ is the mean energy; $r_{i}$ is a random number with a standard normal distribution.

To calculate $V_{\text {ind }}$ we have used the same method used by Bane [3], i.e. binning the macroparticles in $z$ without smoothing. Then the voltage $V_{\text {ind }}$ induced by the beam is given by

$$
V_{\text {ind }}(z)=-e \sum_{i} N_{k} W\left(z-z_{k}\right),
$$

where $N_{k}$ is number of particles in the $k^{\text {th }}$ bin and $W(z)$ is the Green function wake ${ }^{\circledR}$ eld. Other methods of ${ }^{\circledR}$ nding $V_{\text {ind }}$ [4] give smoother results for a given number of macroparticles, but are more CPU-intensive.

For this study, we used a resonator wake ®eld:

$$
W(z)=\frac{\omega_{0} R}{Q} e^{-\frac{k_{0} z}{2 Q}}\left[\cos \left(k_{1} z\right)-\frac{\sin \left(k_{1} z\right)}{\sqrt{4 Q^{2}-1}}\right]
$$

where $R$ is shunt resistance, $\omega_{0}$ the resonant frequency, $k_{0}=$ $\omega_{0} \sigma_{z} / c$ is roughly the bunch length in units of the vacuum chamber size, and $k_{1}=k_{0} \sqrt{1-1 / 4 Q^{2}}$.

In order to be able to relate the results of this paper with earlier work [6], [7], it is convenient to use as intensity the dimensionless parameter $I=e N \omega_{0}(R / Q) /\left(V_{\mathrm{rf}}^{\prime} \sigma_{z 0}\right)$.

The radiation damping usually takes tens or even hundreds of synchrotron oscillations. However, such long damping times require in general too much CPU time to simulate easily. Fortunately, the damping rate does not play a signi®cant role in instabilities which are fast compared with synchrotron motion. This is the regime of the present study. To optimize computation time versus simulation accuracy, we used arti®cial radiation damping times on the order of 5 to 10 times the synchrotron oscillation period.

We found that a reasonable accuracy is achieved with as few as 5,000 macroparticles. This depends upon the wake ®eld being fairly smooth: many times more macroparticles are required for wake ®elds which have many oscillations in one bunch length.[3]

For our analysis we have chosen a resonator wake $® e l d$ with a quality factor $Q=1$ and bunch length parameter $k_{0}=0.5$. The radiation damping time $\tau_{e}$ was set to 500 turns and other parameters $V_{\mathrm{rf}}^{\prime}, \alpha, E_{0}$ in eqn. 2 have been chosen to obtain a synchrotron period of 100 turns.

\section{B. Results}

We start with a large emittance and allow the beam to damp. At low intensities the beam relaxes to a thermodynamically stationary distribution which is well described by the Haissinski equation [5]. However when the intensity increases it takes more time for particles to reach a thermodynamical equilibrium particularly when this distribution has a two-peak line density pro®le. In the case $k_{0}=0.5$ the second peak in the line density appears approximately at $I=10$. This is near the stability threshold found by solving the Vlasov equation [7]. See Fig. 5.

The region close to threshold is dif®cult to model because of the slow growth rate of the instability. Above approximately 

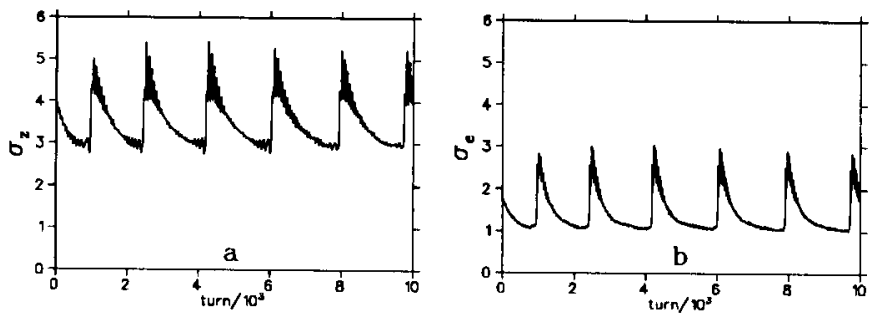

Figure. 1. RMS bunch length (a) and rms energy spread (b) in case of resonator impedance $\left(Q=1, k_{0}=0.5\right)$ at $I=30$. Radiation damping time is $\tau_{e}=5 T_{s}$.

$I=20$, the sawtooth instability becomes apparent. As intensity is raised, the sawtooth periodicity also increases. A typical example showing rms bunch length and energy spread is in $® g .1$ for $I=30$. At very high intensity, the behaviour becomes irregular: the case of $I=45$ is shown in ${ }^{\circledR} \mathrm{g} .2$.
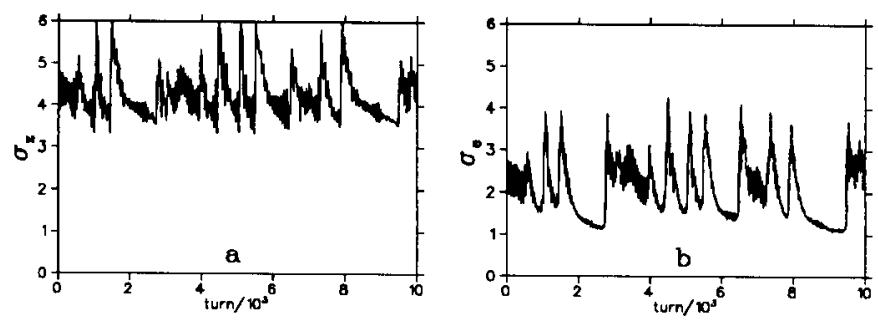

Figure. 2. RMS bunch length and energy spread for the same parameters as Fig. 1, except that $I=45$.

We found that the sawtooth repetition rate is mainly determined by the diffusion process and not by radiation damping. To illustrate this point, the case of a 10/3 times stronger radiation damping is shown on Fig. 3. Comparing Fig. 3 with Fig. 1, one can see that the sawtooth frequency has not changed.
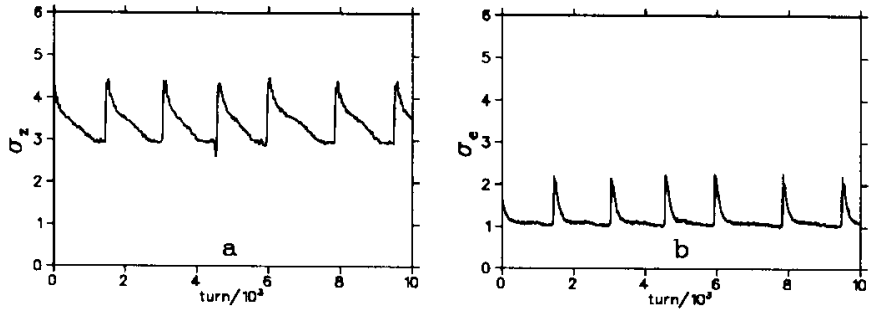

Figure. 3. RMS bunch length and energy spread for an increased damping rate: $I=30$ and $\tau_{e}=1.5 T_{s}$. Compare with Fig. 1.

A complete cycle corresponding to one 'tooth' is shown on Fig. 4:

- $\mathbf{a} \rightarrow \mathbf{b}$ : The downstream cloud damps down (about 5 synchrotron oscillations).

- $\mathbf{b} \rightarrow \mathbf{c}$ : Diffusion populates the second peak until it is approximately equal to the ${ }^{\circledR}$ rst (about 30 synchrotron oscillations). Note that the two peaks have started to move toward each other and a third peak is already beginning to form.
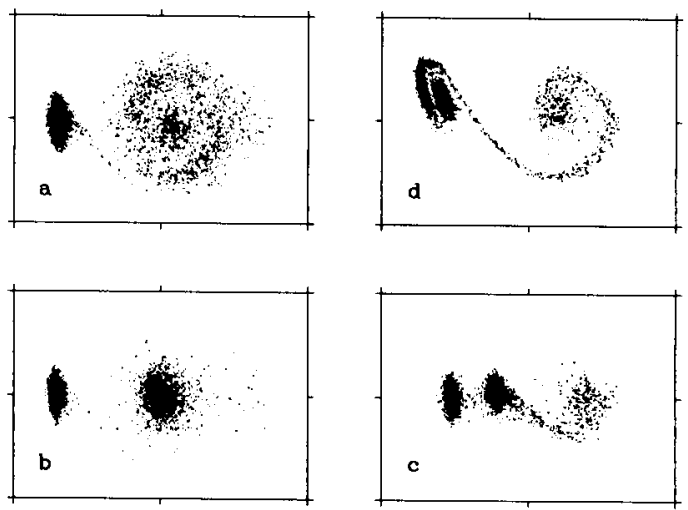

Figure. 4. A complete cycle of the sawtooth instability for the case shown in Fig. 1: $I=30$ and $\tau_{e}=5 T_{s}$. The time sequence is anticlockwise.

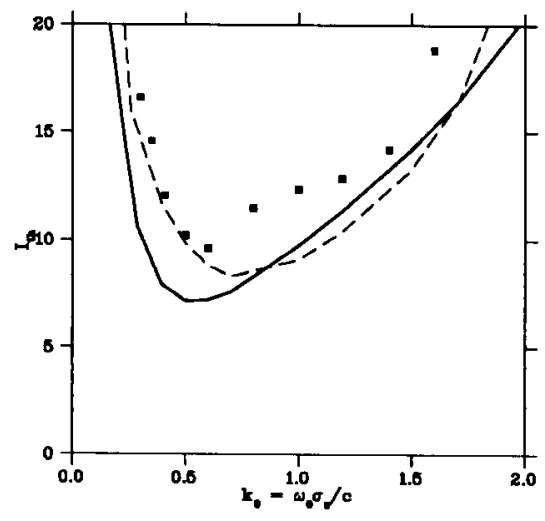

Figure. 5. Threshold intensity vs. bunch length parameter $k_{0}$ in the case of a broad-band $(Q=1)$ resonator. The points and different curves are the results of different calculations. See [7]

- c $\rightarrow$ d In about $1 / 3$ of a synchrotron period the two main sub-bunches collapse together.

- $\mathbf{d} \rightarrow \mathbf{a}$ The combined bunch throws out a large cloud of particles as it executes large synchrotron oscillations (less than a synchrotron period).

The sawtooth behaviour was most clearly seen in the region $0.4<k_{0}<0.6$. For $k_{0}<0.4$, the diffusion process was too slow. For $k_{0}>0.6$, where the threshold intensity increases with bunch length (Fig. 5), sawtooth behaviour is not seen either; instead, the bunch length oscillates chaotically.

\section{Analysis}

Qualitatively, the instability can be understood by considering the wake of an extremely short bunch (Fig. 6, upper). In order for the energy lost by the bunch to the wake ${ }^{8}$ eld to be compensated by the rf cavities, the rf waveform (here drawn as a straight line) must intersect the wake voltage at half the maximum. This is the location of the centre of this very short bunch, and is of course a stable ${ }^{\circledR} x e d$ point. Situations for various $r f$ voltage values can be considered by pivoting the rf waveform (line) about this point, as indicated in Fig. 6. Situations with differing beam intensities can be simulated in the same way, since amplifying the wake ®eld 

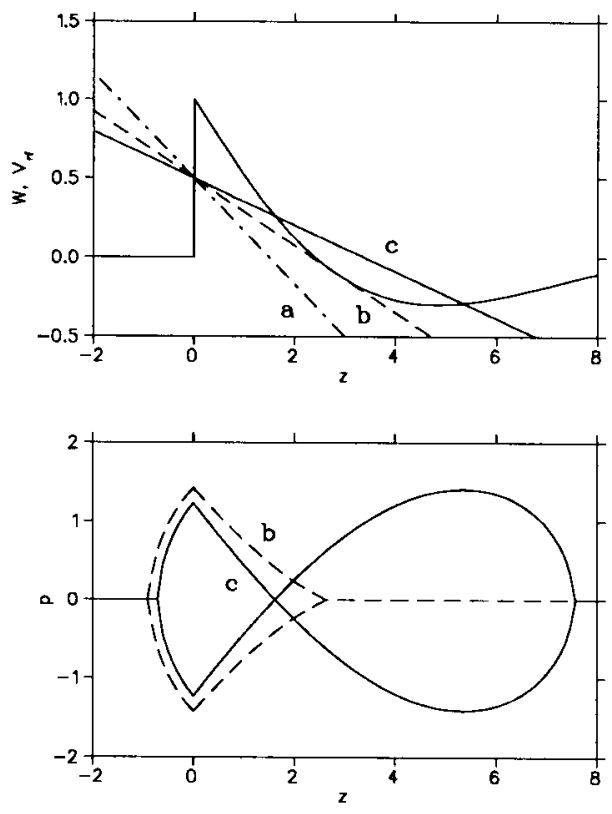

Figure. 6. Green function wake ®eld (upper window), including $3 \mathrm{rf}$ waveform slopes; (a) is stable, (b) is just above threshold, and (c) is in the sawtooth regime. Looking from left to right, there is a stable ${ }^{\circledR} x e d$ point if the wake ${ }^{\circledR}$ eld crosses the rf waveform from below, and an unstable $® x$ xed point if it crosses from above. The separatrices created by the wake ®elds corresponding to cases (b) and (c) have been plotted in the lower window. In case (a), there is only one stable $® x$ xed point so the wake ${ }^{\circledR}$ eld does not create a separatrix. Note that these curves are for a Green function wake and therefore are only suggestive. Any accumulation of a ${ }^{\circledR}$ nite charge density will deform the separatrices.

has the same effect on the diagram as reducing the rf slope. At low intensity or large rf voltage, there is only the one ${ }^{\circledR} x e d$ point. At high intensity or low rf voltage, the wake ${ }^{\circledR}$ eld intersects the rf waveform at three points; there is an unstable $® x$ xed point behind the bunch, and a stable one farther along. Separatrices created by the extra ®xed points are shown in the lower window in Fig. 6.

Because of the random excitation due to emission of synchrotron radiation, particles can diffuse through the unstable ®xed point and collect at the downstream stable ${ }^{\circledR} x e d$ point. These particles begin to create their own wake, and will have to move forward as they lose energy to their own wake ®eld. At the same time, the remaining particles in the head sub-bunch will move backwards as they decrease in number and no longer need as large energy gain from the rf ®eld. At some point, the potential barrier between the two sub-bunches becomes small enough that the diffusion turns into an avalanche and the sub-bunches suddenly coalesce. The resulting bunch is over-dense and at the wrong phase with respect to the needed energy gain. It begins to execute a large synchrotron oscillation, while beginning again to lose particles to diffusion. This results in a large cloud of particles and a large rms bunch length and energy spread. The cloud condenses again at the downstream stable $®$ xed point and diffusion continues.

\section{Conclusion}

We have developed a qualitative picture of the sawtooth instability. The wake ®eld creates its own unstable and stable ${ }^{\circledR} x e d$ points, particles diffuse to the second @xed point, and then the resulting second sub-bunch collapses into the head sub-bunch. The sawtooth frequency is therefore determined not primarily by radiation damping, but by a subsequent diffusion process.

The sawtooth effect is most readily seen when the bunch length is comparable with the wake ®eld length. Qualitatively quite different behaviours can be seen when the bunch is either short or long compared with the wake. In the former case, for example, the two sub-bunches can sometimes pass through each other instead of collapsing, thus leading to a sustained quadrupole oscillation. This may be the type of behaviour seen in LEP [8]. These regimes as well as other types of wake ®elds are still under investigation.

\section{References}

[1] P. Krejcik et al., High Intensity Bunch Length Instability in the SLC Damping Rings Proc. PAC93 (Washington D.C.) p. 3240.

[2] G. Rakovsky and L.R. Hughey, SURF's up at NBS: a Progress Report IEEE Trans Nucl. Sci. NS-26 (1979) p. 3845.

[3] K.L.F. Bane and K. Oide, Simulations of the Longitudinal Instability in the SLC Damping Rings, Proc. PAC93 (Washington D.C.) p. 3339.

[4] R. Siemann, Computer Simulation of Bunch Lengthening in SPEAR Nucl. Instr. Meth., 203 (1982) p. 57.

[5] J. Haissinski, Exact Longitudinal Equilibrium Distribution of Stored Electrons in the Presence of Self-Fields Il Nuovo Cimento 18B (1973) p. 72.

[6] K. Oide and K. Yokoya, Longitudinal Single Bunch Instability in Electron Storage Rings KEK Preprint 90-10.

[7] M. D'yachkov and R. Baartman, Method for Finding Bunched Beam Instability Thresholds Proc. EPAC94 (London) p. 1075.

[8] D. Brandt, K. Cornelis and A. Hofmann, Experimental Observations of Instabilities in the Frequency Domain at LEP Proc. EPAC92 (Berlin) p. 345. 